\title{
CNO Abundances of BA-Type Supergiants
}

\author{
M. Firnstein* and N. Przybilla \\ Dr. Remeis-Sternwarte, Bamberg \\ E-mail: firnsteinesternwarte.uni-erlangen.de, \\ przybilla@sternwarte.uni-erlangen.de
}

Massive BA-type supergiants are among the visually brightest stars in galaxies with active star formation. As such they are versatile tools for studies of stellar and galactochemical evolution. Moreover, they can act as distance indicators for the calibration of the cosmological distance scale. In the present work abundance patterns of the light elements helium, carbon, nitrogen and oxygen are investigated in several Galactic BA-type supergiants in the mass range between 8 and $18 M_{\odot}$. Based on high-resolution and high-S/N Echelle spectra obtained with FOCES on the Calar Alto $2.2 \mathrm{~m}$ telescope, model atmosphere analyses are performed using state-of-the-art non-LTE spectrum synthesis. Stellar parameters and chemical abundances are determined with high accuracy. This gives tight observational constraints on the evolutionary status of the stars. Objects evolving from the main sequence to the red supergiant stage and those on a blue loop can be distinguished by their mixing signature (pure rotational vs. first dredge-up). The most sensitive tracer of nuclear processed matter, the N/C ratio, indicates a higher mixing efficiency than predicted by current evolution models of rotating stars with mass-loss.

International Symposium on Nuclear Astrophysics - Nuclei in the Cosmos - IX

June 25-30 2006

CERN, Geneva, Switzerland

\footnotetext{
* Speaker.
} 


\section{CNO-Abundances and the Evolution of Massive Stars}

BA-type supergiants are among the visually brightest massive stars, allowing for a mapping of the spatial distribution of elemental abundances throughout galaxies and therefore a determination of abundance gradients. They can be versatile indicators for studies of stellar and galactic evolution as well as in the cosmological context. Comprehensive quantitative understanding of BA-type supergiants in the Milky Way is an essential step on the way to use this potential.

An introduction to current stellar evolution models of massive stars can be found in [母] or [1]. Stars on the main sequence (MS) - during the longest part of their life - burn hydrogen to helium in their cores. For massive stars with their high core temperature and pressure the most efficient process for this turns out to be the CNO-bi-cycle. While the actual elements carbon, nitrogen and oxygen only function as catalysts, meaning no net production or loss occurs during a cycle, their relative numbers change over the period of hydrogen burning. The reaction ${ }^{14} \mathrm{~N}(p, \gamma)^{15} \mathrm{O}$ is a bottleneck, because it is the slowest by far. The abundance of the stable nitrogen isotope increases while the abundances of the stable carbon and oxygen isotopes decrease, with the most abundant oxygen isotope ${ }^{16} \mathrm{O}$ being transformed more slowly in the minor NO-branch of the cycle.

Stellar spectra trace abundances only of the photospheric layers. In order for processed matter to be recognisable, it has to travel the long way from the centre of the star to the outer layers. Massive stars on the main sequence possess no convective envelope like the Sun, ruling out convection as transport mechanism. However, slower mixing mechanisms exist, like rotation-induced meridional circulation or shear mixing (caused by differential rotation). Their efficiency increases with both rotational velocity and mass of the star and can also depend on the magnetic field strength.

BA-type supergiants have already left the main sequence, their progenitors being OB-stars. Their envelope is still in radiative equilibrium, so that no major changes in the abundance patterns are expected during the short post-MS phase, should they have evolved directly from the MS. For less-massive supergiants another scenario is possible, the so called blue loop. Such objects have already reached the red supergiant stage, but eventually evolve back into a blue supergiant. During the red supergiant phase, their envelope has been fully convective and intense mixing has taken place (first dredge-up). This strengthens the signature of CNO-processed material in the photosphere considerably, allowing to distinguish them from the other class of supergiants.

\section{Observations and Quantitative Spectral Analysis}

Quantitative spectroscopy with high precision is required to derive information on the evolutionary status of BA-type supergiants. On the observational side high-resolution and high-S/N spectra have been obtained using the Echelle spectrograph FOCES at the Calar Alto 2.2m telescope. Therefore objects in the solar neighbourhood - preferably associated to open clusters - of spectral types B5-A3 and luminosity classes Ib to Ia have been chosen, in order to cover this part of the HRD as completely as possible. To extract the abundance information from the spectra, they are compared to synthetic spectra using the fit routine FITPROF (see [6] for a description). Previous quantitative analyses of Galactic BA-SGs are mostly based on standard LTE techniques, which provide only limited accuracy. Recently, we introduced a NLTE spectrum synthesis tech- 


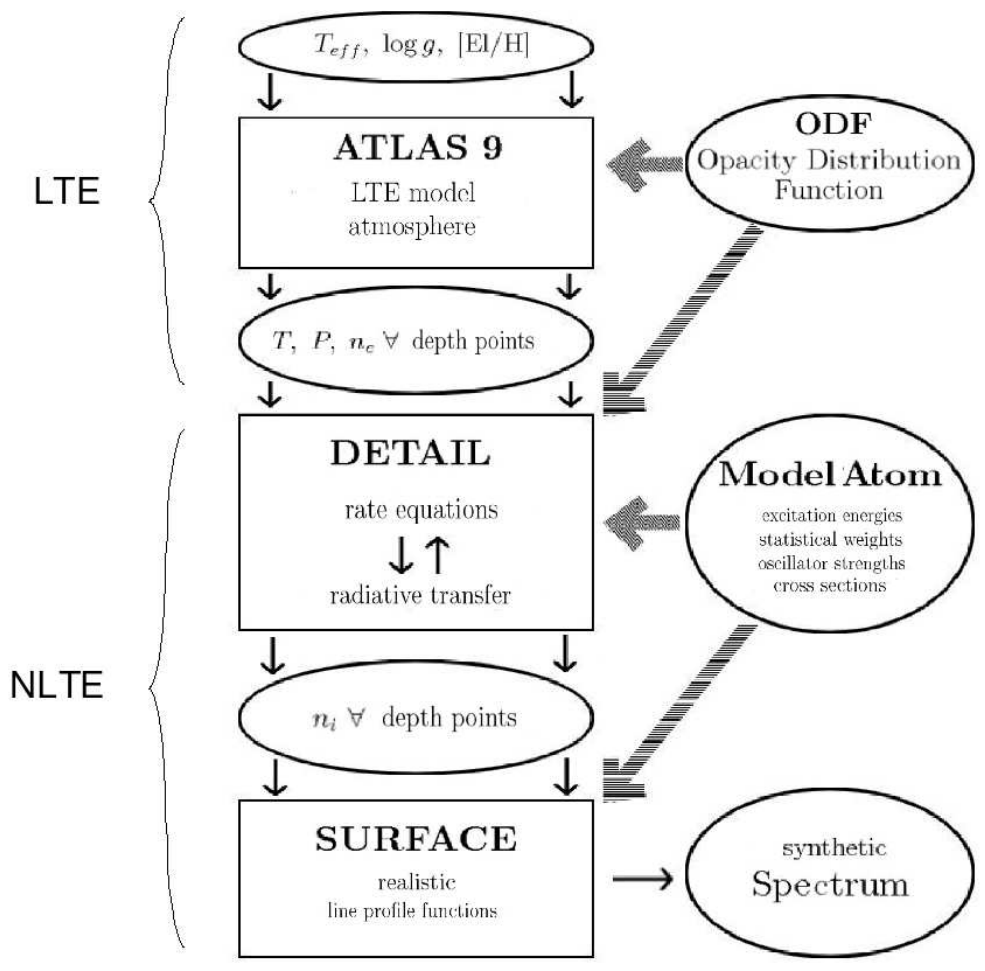

Figure 1: Overview of the model calculation process

nique that allows for high-precision analyses of BA-SGs ([8]). NLTE line-formation computations are performed with DETAIL and SURFACE on the basis of hydrostatic line-blanketed LTE model atmospheres (ATLAS9) in plane-parallel geometry, see Fig. 1 for an overview. This method takes new state-of-the-art model atoms for $\mathrm{H}, \mathrm{He}$ I, C I/II, N I/II, O I and Mg I/II into account, based on accurate atomic data and modern line-broadening theories. This allows for a highly accurate determination of metal abundances with low statistical scatter (10-20\% on the $1 \sigma$-level) and leads to highly consistent abundances for individual stars ([9]) as well as for a larger sample of objects (this work).

The stellar parameters are determined via line-profile fits to the Stark-broadened hydrogen Balmer and $\mathrm{He}$ I lines, as well as ionization equilibria such as $\mathrm{Mg} \mathrm{I} / \mathrm{II}$ for cooler stars and $\mathrm{N} \mathrm{I/II} \mathrm{for}$ hotter stars of the sample. The method is described by [8].

While neither of the criteria alone allows the unambiguous determination of effective temperature $T_{\text {eff }}$ and surface gravity $\log g$, a combination of them does. Problems may arise for $\mathrm{H} \alpha$, as stellar wind effects are neglected in our modelling, the effects being strongest in the most massive and therefore most luminous supergiants.

High accuracy in the parameter determination is required in order to derive chemical abundances with high precision. Not only abundance, $T_{\text {eff }}$ and $\log g$ influence the line strength, but also the microturbulence and the helium content. After determining all these parameters by iteration we can achieve accuracies in chemical abundances of better than 0.1 dex, depending on the number of available lines and the quality of the spectrum. 


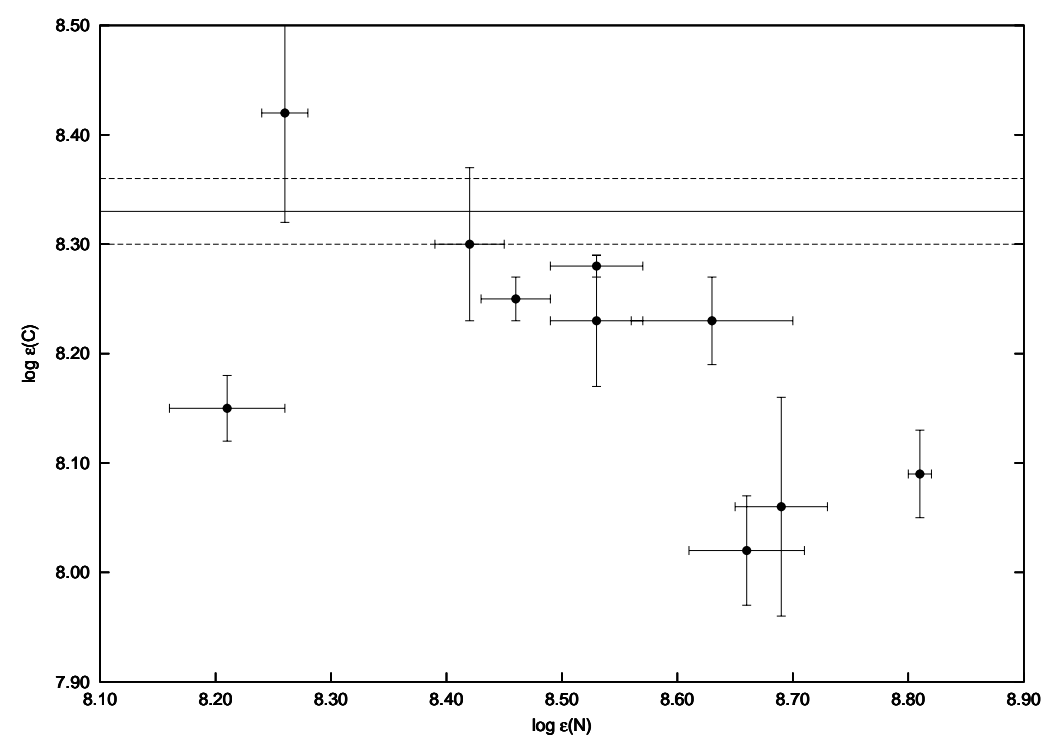

Figure 2: Carbon abundances as a function of the nitrogen abundance for the 10 stars of the sample, where $\log \varepsilon(\mathrm{X})=\log \left(N_{\mathrm{X}} / N_{\mathrm{H}}\right)+12$. They are compared to the mean value $8.33 \pm 0.03$ obtained by 㠶 from carbon abundances in $6 \mathrm{~B}$-dwarfs and giants

\section{Results and Conclusions}

The abundance data for the 10 analysed BA-Type supergiants show some interesting results. While magnesium and oxygen abundances show only a small scatter, the expected trends for carbon and in particular nitrogen become apparent (see Fig. 2).

In combination with a precise determination of the stellar parameters the abundance analysis allows comparisons with stellar evolution models. Apparently the N/C ratios are generally larger than predicted by theory, and significantly larger than constrained from observation before ([10],[11]: we have four objects in common with those studies). More recent stellar evolution computations accounting for the interplay of rotation and magnetic fields, as described by [3] , promise to resolve these discrepancies as they show a much higher efficiency for chemical mixing. A possibly even better solution provide newer measurements of the ${ }^{14} \mathrm{~N}(p, \gamma){ }^{15} \mathrm{O}$ cross section ([2]), that reduce previous estimates of the reaction rate by a factor of 2 . High N/C ratios also particularly occur in regions where blue loops are predicted, implying that these objects have undergone the first dredge-up (see Fig. 3). However, the blue loops may need to extend further to the blue than predicted by the current models.

\section{References}

[1] A. Heger, N. Langer, S.E. Woosley, Presupernova Evolution of Rotating Massive Stars. I. Numerical Method and Evolution of the Internal Stellar Structure, 2000, ApJ, 528, 368

[2] A. Lemut et al., First measurement of the ${ }^{14} \mathrm{~N}(p, \gamma){ }^{15} \mathrm{O}$ cross section down to $70 \mathrm{keV}, 2006$, Physics Letters B, 634, 483

[3] A. Maeder, G. Meynet, Stellar evolution with rotation and magnetic fields. III. The interplay of circulation and dynamo, 2005, A\&A, 440, 1041 [astro-ph/ 0506347 ] 


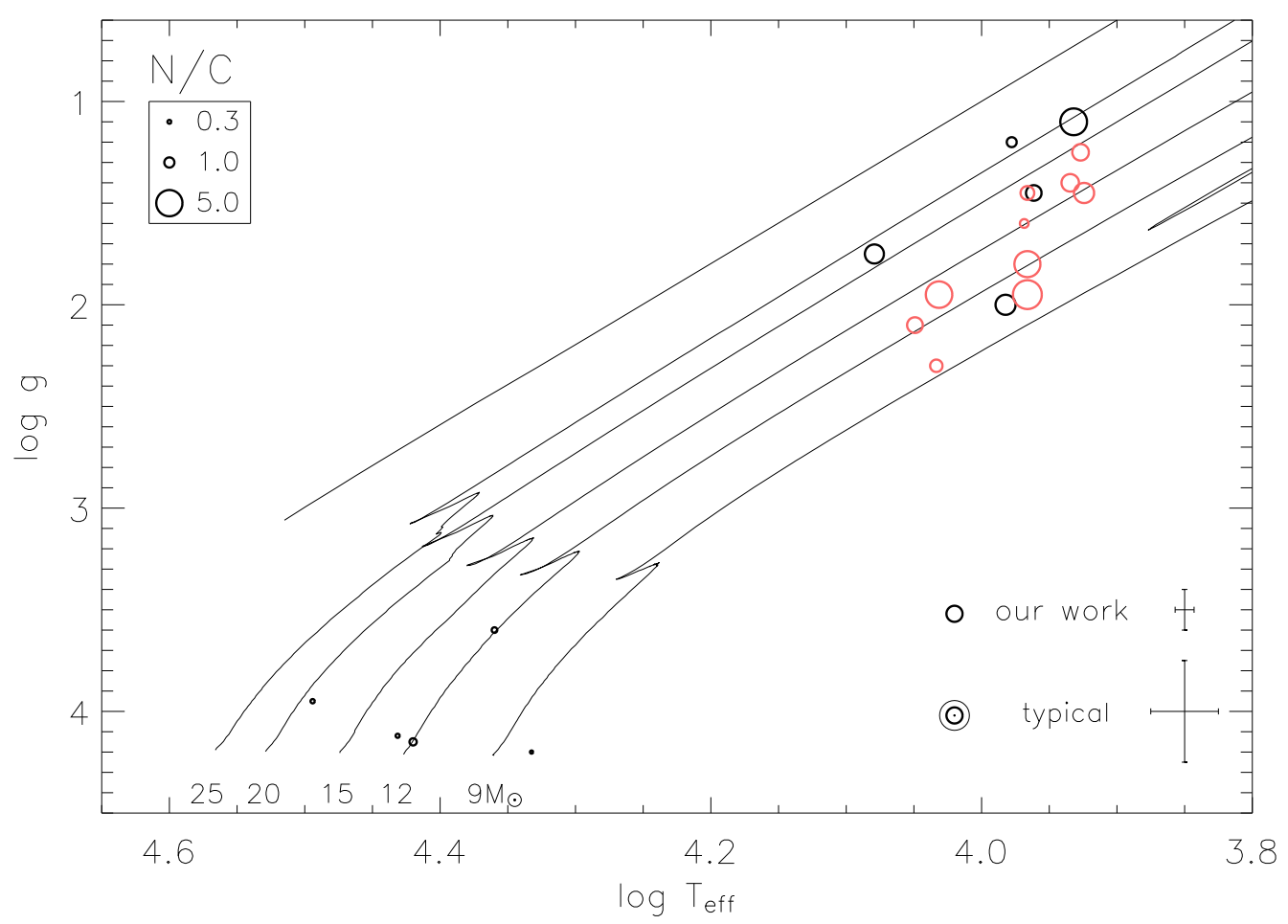

Figure 3: Data for objects analysed in this work (coloured circles) are compared to evolution tracks from [5] for rotating $\operatorname{stars}\left(v_{\text {rot }}=300 \mathrm{~km} / \mathrm{s}\right)$. The models start with a N/C ratio of 0.3 on the main sequence and predict ratios of 0.7 to 1.7 for BA-type supergiants in the mass range of 9 to $25 M_{\odot}$ evolving redwards and 1.6 to 2.6 for stars of 9 to $12 M_{\odot}$ on a blue loop, while our findings for 10 BA-type supergiants show N/C ratios between 0.81 and 6.16. Also displayed are additional results for BA-type supergiants from [8] and B-dwarfs and giants from [ד], all analysed in a homogenous way.

[4] A. Maeder, G. Meynet, Stellar evolution with rotation, 2000, ARA\&A, 38, 143

[5] G. Meynet, A. Maeder, Stellar evolution with rotation. X. Wolf-Rayet star populations at solar metallicity, 2003, A\&A, 404, 975 [astro-ph/ 0304069 ]

[6] R. Napiwotzki, P.J. Green, R.A. Saffer, A Comparative Study of the Mass Distribution of Extreme-Ultraviolet-selected White Dwarfs, 1999, ApJ, 517, 399 [astro-ph/9901027]

[7] M.F. Nieva, N. Przybilla, C II Abundances in Early-Type Stars: Solution to a Notorious Non-LTE Problem, 2006, ApJ, 639, L39 [astro-ph/ 0602342]

[8] N. Przybilla, K. Butler, S.R. Becker, R.P. Kudritzki, Quantitative spectroscopy of BA-type supergiants, 2006, A\&A, 445, 1099 [ast ro-ph/ 0509669 ]

[9] F. Schiller, N. Przybilla, Quantitative Spectroscopy of Deneb, 2006, PoS(NIC-IX)174

[10] K.A. Venn, CNO Abundances and the Evolutionary Status of Galactic, A-Type Supergiants, 1995, ApJ, 449, 839

[11] K.A. Venn, N. Przybilla, New Nitrogen and Carbon in AF-supergiants, 2003, ASP Conf. Ser, 304, 20 